\section{Síndrome metabólico e inflamación en adultos. Un estudio poblacional}

\author{
PATRICIA BUSTOS, BERNARDITA ROSAS, PABLO ROMÁN ${ }^{a}$, \\ JUAN VILLAGRÁN ${ }^{\mathrm{a}}$, HUGO AMIGO ${ }^{\mathrm{b}}$
}

\section{Association of $\mathrm{C}$ reactive protein levels with metabolic syndrome in adults: a population-based study}

Background: Metabolic syndrome is highly prevalent among adults in Chile and represents a health risk. Aim: To determine the relationship between metabolic syndrome (MetS) and its components, with $C$ reactive protein levels $(C R P)$ as an inflammation marker. Material and Methods: The population studied consisted of 736 individuals born in a hospital from Valparaiso Region, aged between 32-38 years at the time of the study. MetS was identified according to the Adult Treatment Panel (ATP) III guidelines and inflammation was measured using ultra-sensitive CRP. This parameter was classified as normal for values from 0 to $3 \mathrm{mg} / \mathrm{L}$, high for values from 3.01 to $10 \mathrm{mg} / \mathrm{L}$ and very high for values $>10 \mathrm{mg} / \mathrm{L}$. Results: Median CRP was in the normal range $(1.9 \mathrm{mg} / \mathrm{L}$, interquartile range 0.7-5.2) and was higher among women than men (2.2 and $1.4 \mathrm{mg} / \mathrm{L}$ respectively, $p<0.01)$. Twenty seven percent of participants had MetS. One-fourth had high blood glucose values, one-third had high triglyceride levels and $28 \%$ had blood pressure values over those established as normal in MetS. Elevated waist circumference (WC) and low HDL cholesterol were found among almost 50\% of participants. A relationship between MetS and high CRP was only found among men with an Odds ratio (OR) of 2.04 (95\% confidence intervals (CI): 1.11-3.73). The same association was observed for high triglyceride levels with an OR of 2.02 (CI: 1.17-3.49) and high WC with an OR of 3.89 (CI: 2.067.36). Among women, the only relationship observed was between abdominal obesity and very high CRP with an OR of 2. 65 (CI: 1.20-5.84). Conclusions: Metabolic syndrome, high triglyceride levels, and abdominal obesity were associated with inflammation only in men.

(Rev Med Chile 2016; 144: 1239-1246)

Key words: Adult; C-Reactive Protein; Metabolic Syndrome X.

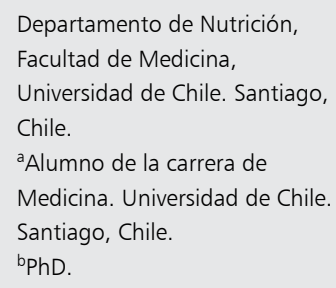

Correspondencia a:

Dra. Patricia Bustos

Independencia 1027. Santiago,

Chile.

Teléfono: 227986213

pbustos@med.uchile.cl
S e ha señalado que las enfermedades crónicas no trasmisibles han aumentado su frecuen$\mathrm{cia}^{1-3}$. También se ha indicado que la inflamación participa en la fisiopatología de varias de estas enfermedades ${ }^{4,5}$, por lo que es importante conocer su asociación para sugerir posibles alternativas de prevención, tratamiento y control.

El síndrome metabólico, patología que agrupa distintos factores de riesgo cardiovascular, com- promete a $27,5 \%$ de las personas entre 25 y 44 años en el país, es más frecuente en varones y tiende a aumentar con la edad ${ }^{6}$. La obesidad central es uno de sus constituyentes, asociándose a numerosas consecuencias y condiciones agregadas, entre ellas, a un estado de inflamación crónica.

Se ha indicado que la proteína $\mathrm{C}$ reactiva que se sintetiza a nivel hepático, en respuesta a enfermedades inflamatorias o infecciones agudas, 
cáncer, quemaduras o post cirugía ${ }^{7,8}$, también está crónicamente aumentada en las personas obesas, indicándose que la inflamación es el mecanismo que potencialmente une la obesidad con el riesgo cardio-metabólico ${ }^{9,10}$. Menos información hay de la asociación de inflamación con síndrome metabólico.

La rápida transición socio-demográfica, alimentaria y nutricional que ha ocurrido en el país en las últimas décadas, se ha acompañado de un aumento de la obesidad y del síndrome metabólico, que se inician cada vez más tempranamente $^{11-13}$. El que los factores de riesgo de enfermedades cardiovasculares se asocien a inflamación en edades más tempranas que cuando se presentan los eventos que causan discapacidad y muerte, permitiría entregar elementos para realizar vigilancia epidemiológica y potenciar programas dirigidos a la prevención y control de enfermedades crónicas en atención primaria. El objetivo de este trabajo fue determinar la asociación entre síndrome metabólico y también de sus componentes con un marcador de inflamación, como la proteína $C$ reactiva ultra sensible (PCRus) en adultos, en un estudio poblacional, en personas que viven en comunas semi-rurales del país.

\section{Material y Métodos}

Este estudio, de corte transversal, evaluó entre los años 2010 y 2012, a 736 personas, que participan en un seguimiento de nacidos entre 1974 y 1978 en el Hospital de Limache, Región de Valparaíso, Chile y que al momento del estudio tenían entre 32 y 38 años.

La información se obtuvo a través de encuestas socioeconómicas, de salud, mediciones antropométricas y exámenes de laboratorio. La presión arterial se midió dos veces, con un manómetro digital, con la persona en reposo. Los análisis de esta variable se realizaron utilizando el promedio de las dos mediciones. Se consideró valores de presión elevados si estaban $\geq 130 / 85 \mathrm{mmHg}$ o si la persona estaba con tratamiento antihipertensivo. El perímetro de cintura se midió con una huincha flexible, en el punto medio entre la cresta iliaca y la última costilla, considerándose obesidad abdominal valores $>88 \mathrm{~cm}$ en mujeres y $>102$ $\mathrm{cm}$ en hombres. En muestra de sangre en ayunas se determinaron niveles plasmáticos de glicemia, colesterolemia y trigliceridemia. Se consideró glicemia elevada cifras $>100 \mathrm{mg} / \mathrm{dL}$; trigicéridos elevados cifras $>150 \mathrm{mg} / \mathrm{dL}$ y cifras bajas de colesterol HDL en varones $<40 \mathrm{mg} / \mathrm{dL}$ y $<50 \mathrm{mg} /$ $\mathrm{dL}$ en mujeres. Se definió síndrome metabólico al conjunto de 3 o más de estos componentes ${ }^{14}$. El nivel socioeconómico (NSE) se utilizó como variable de ajuste, estimándose mediante una matriz construida con la ocupación y escolaridad del jefe de hogar ${ }^{15}$.

La inflamación se midió a través de la PCR ultra sensible (que permite medir niveles que no son detectables con otros métodos) categorizándola en: normal (de 0 a $3 \mathrm{mg} / \mathrm{L}$ ), elevada (de 3,01 a 10) y muy alta $(>10 \mathrm{mg} / \mathrm{L})$, clasificación que es una adaptación de la sugerida por Yeh y Willerson ${ }^{16}$.

Las encuestas, la evaluación clínica y la obtención de sangre fueron realizadas por nutricionistas o enfermeras capacitadas, con estándares comunes y permanentemente supervisadas.

Inicialmente se describió la población en estudio a través de medidas centrales (mediana y percentiles 25 y 75) y se hicieron asociaciones bivariadas con las variables de interés. Posteriormente, se realizó un análisis de regresión logística multinomial para estudiar la asociación entre síndrome metabólico y de cada uno de sus componentes con el marcador de inflamación mencionado en sus categorías elevada y muy alta, considerando como grupo de referencia los valores clasificados como normales, estimándose los OR con intervalos de confianza (95\% IC). Estos modelos fueron ajustados por potenciales confusores (sexo y nivel socioeconómico), explorándose la existencia de interacciones.

Este estudio fue aprobado por el Comité de Ética de la Facultad de Medicina de la Universidad de Chile, todos los participantes firmaron un consentimiento informado previo al ingreso al estudio.

\section{Resultados}

La mediana de edad de la población estudiada fue de 35,2 años; 9\% fue clasificado en el NSE bajo y la mitad estuvo en los niveles medio y medio alto, destacando que en esta muestra no hubo personas clasificadas en el nivel alto. La mediana de IMC estuvo desplazada hacia el exceso de peso, especialmente en las mujeres. Las medianas de 
glicemia, triglicéridos y presión arterial estuvieron en valores normales y fueron significativamente mayores en hombres; el perímetro de cintura, como era de esperar, fue mayor en hombres. Si bien los niveles de colesterol HDL no difirieron por sexo, los valores encontrados en mujeres estaban bajo los valores recomendados de normalidad. La PCRus estuvo en un rango normal bajo, con valores significativamente mayores en mujeres (Tabla 1).

El 27\% de las personas presentaron SM, levemente más frecuente en las mujeres. Un cuarto de la población tuvo la glicemia elevada, un tercio los triglicéridos aumentados y $28,4 \%$ la presión arterial en valores mayores a los establecidos para $\mathrm{SM}$, todas estas cifras fueron mayores en hombres. El perímetro de cintura aumentado y los HDL bajos tuvieron prevalencias mayores, que llegaron a comprometer a la mitad de la población estu- diada y, a diferencia de los anteriores, las mujeres los presentaron alterados con mayor frecuencia (Tabla 2).

Un tercio de la población tenía valores de PCRus elevados y algo menos de $7 \%$ presentó cifras muy altas, con valores mayores en las mujeres. $\mathrm{Al}$ analizar las categorías de PCRus elevada y muy alta y su relación con SM, se pudo comprobar que en hombres, hubo mayor frecuencia de valores de PCRus elevados en aquellos que tenían SM $(\mathrm{p}=0,049)$; en mujeres no se observó esto $(\mathrm{p}=0,775)$ (Tabla 3).

Debido a que se verificó una interacción entre SM y sexo, para controlar este efecto, se elaboraron modelos multinomiales separados para hombres y mujeres.

En los hombres se observó una asociación significativa de SM y PCRus elevada, alcanzando una OR de $2(p=0,021)$. En relación a los componen-

Tabla 1. Características generales de la población estudiada según sexo

\begin{tabular}{|c|c|c|c|c|}
\hline & $\begin{array}{c}\text { Total } \\
(n=751)\end{array}$ & $\begin{array}{l}\text { Hombres } \\
(n=260)\end{array}$ & $\begin{array}{l}\text { Mujeres } \\
(n=491)\end{array}$ & $\mathbf{p}$ \\
\hline Edad (años) & $\begin{array}{c}35,2 \\
(34,1-36,5)\end{array}$ & $\begin{array}{c}35,5 \\
(34,3-36,7)\end{array}$ & $\begin{array}{c}35,0 \\
(34,0-36,4)\end{array}$ & 0,008 \\
\hline \multicolumn{5}{|l|}{ Nivel socioeconómico (\%) } \\
\hline Bajo & 8,8 & 10,5 & 7,9 & \multirow{3}{*}{0,510} \\
\hline Medio bajo & 42,5 & 41,5 & 43,0 & \\
\hline Medio y medio alto & 48,7 & 48,1 & 49,1 & \\
\hline IMC $\left(\mathrm{kg} / \mathrm{m}^{2}\right)$ & $\begin{array}{c}27,8 \\
(25,0-31,1)\end{array}$ & $\begin{array}{c}27,3 \\
(24,5-30,1)\end{array}$ & $\begin{array}{c}28,2 \\
(25,2-31,5)\end{array}$ & 0,003 \\
\hline Glicemia (mg/dL) & $\begin{array}{c}92 \\
(84-99)\end{array}$ & $\begin{array}{c}95 \\
(88-101,7)\end{array}$ & $\begin{array}{c}90 \\
(83-96)\end{array}$ & 0,0001 \\
\hline Triglicéridos (mg/dL) & $\begin{array}{c}116 \\
(82-173)\end{array}$ & $\begin{array}{c}124,5 \\
(114-135)\end{array}$ & $\begin{array}{c}115,2 \\
(106-125)\end{array}$ & 0,0001 \\
\hline Perímetro de cintura $(\mathrm{cm})$ & & $\begin{array}{c}93,5 \\
(87-100)\end{array}$ & $\begin{array}{c}91,2 \\
(82,7-99,8)\end{array}$ & \\
\hline $\mathrm{HDL}(\mathrm{mg} / \mathrm{dL})$ & & $\begin{array}{c}48,0 \\
(40-56)\end{array}$ & $\begin{array}{c}47,0 \\
(39-54)\end{array}$ & 0,123 \\
\hline $\begin{array}{l}\text { Presión arterial sistólica } \\
(\mathrm{mmHg})\end{array}$ & $\begin{array}{c}119 \\
(108,5-129)\end{array}$ & $\begin{array}{c}124,5 \\
(114-135)\end{array}$ & $\begin{array}{c}115,2 \\
(106-125)\end{array}$ & 0,0001 \\
\hline $\begin{array}{l}\text { Presión arterial diastólica } \\
(\mathrm{mmHg})\end{array}$ & $\begin{array}{c}74 \\
(66,7-81,5)\end{array}$ & $\begin{array}{c}76,0 \\
(69-84,5)\end{array}$ & $\begin{array}{c}72,5 \\
(65,5-80)\end{array}$ & 0,0001 \\
\hline PCRus (mg/L) & $\begin{array}{c}1,9 \\
(0,7-5,2)\end{array}$ & $\begin{array}{c}1,4 \\
(0,6-4,2)\end{array}$ & $\begin{array}{c}2,2 \\
(0,8-5,8)\end{array}$ & 0,001 \\
\hline
\end{tabular}

Valores expresados en medianas y p25-p75. Significancia: prueba de Mann-Whitney para medianas, $\chi^{2}$ para la distribución del NSE. 
tes del SM, los varones que presentaban triglicéridos elevados tuvieron OR de $2,0(\mathrm{p}=0,011)$ y los con perímetro de cintura elevado, un OR cercano a $4(\mathrm{p}=0,0001)$. No se observó asociación entre el resto de los componentes del SM (hipertensión arterial, bajos niveles de HDL o glicemia elevada) con inflamación en grado moderado o muy alta (Tabla 4). En mujeres no se observaron estas asociaciones, con excepción del PC elevado y PCRus muy alta, con OR de 2,65 ( $\mathrm{p}=0,016)$ (Tabla 5).

Tabla 2. Frecuencia del Síndrome metabólico y sus componentes, según sexo

\begin{tabular}{|c|c|c|c|c|}
\hline & Total & Hombres & Mujeres & $\mathbf{p}$ \\
\hline Síndrome metabólico & $\begin{array}{c}27,3 \\
(25,0-31,0)\end{array}$ & $\begin{array}{c}24,7 \\
(19,0-29,0)\end{array}$ & $\begin{array}{c}29,9 \\
(26,0-34,0)\end{array}$ & 0,048 \\
\hline Glicemia elevada & $\begin{array}{c}23,6 \\
(20,5-26,6)\end{array}$ & $\begin{array}{c}30,7 \\
(25,7-37,3)\end{array}$ & $\begin{array}{c}19,0 \\
(15,8-22,8)\end{array}$ & 0,0001 \\
\hline Triglicéridos elevados & $\begin{array}{c}33,9 \\
(30,4-37,3)\end{array}$ & $\begin{array}{c}44,8 \\
(38,6-50,8)\end{array}$ & $\begin{array}{c}27,7 \\
(24,1-32,1)\end{array}$ & 0,0001 \\
\hline Presión arterial elevada & $\begin{array}{c}28,4 \\
(25,0-32,0)\end{array}$ & $\begin{array}{c}40,5 \\
(35,0-47,0)\end{array}$ & $\begin{array}{c}22,0 \\
(18,0-26,0)\end{array}$ & 0,0001 \\
\hline Perímetro de cintura elevado & $\begin{array}{c}46,1 \\
(42,5-49,7)\end{array}$ & $\begin{array}{c}20,5 \\
(15,5-25,4)\end{array}$ & $\begin{array}{c}59,7 \\
(55,3-64,0)\end{array}$ & 0,0001 \\
\hline HDL bajos & $\begin{array}{c}46,9 \\
(43,0-50,0)\end{array}$ & $\begin{array}{c}24,3 \\
(19,0-29,6)\end{array}$ & $\begin{array}{c}58,9 \\
(54,0-63,2)\end{array}$ & 0,0001 \\
\hline
\end{tabular}

Valores expresados en \% e intervalos de confianza.

Tabla 3. Distribución de la PCR en categorías, según sexo y presencia o no de SM

\begin{tabular}{|lcccccc|}
\hline PCRus & \multicolumn{2}{c}{ Total (\%) } & \multicolumn{2}{c|}{ Hombres (\%) } & \multicolumn{2}{c|}{ Mujeres (\%) } \\
& Sin SM & Con SM & Sin SM & Con SM & Sin SM & Con SM \\
Normal & 61,7 & 55,5 & 69,5 & 54,8 & 57,3 & 55,8 \\
$(0-2,9$ mg/L) & $(57,6-65,8)$ & $(48,8-62,2)$ & $(63,1-75,9)$ & $(42,6-67,0)$ & $(52,1-62,5)$ & $(47,8-63,8)$ \\
Elevada & 31,4 & 38,8 & 25,9 & 41,9 & 34,6 & 37,4 \\
$(3-10$ mg/L) & $(27,5-35,3)$ & $(32,2-45,4)$ & $(19,8-32,0)$ & $(29,6-54,2)$ & $(29,6-39,6)$ & $(30,1-45,2)$ \\
Muy alta & 6,8 & 5,7 & 4,6 & 3,2 & 8,2 & 6,7 \\
$(>10$ mg/L) & $(4,7-8,9)$ & $(2,6-8,8)$ & $(1,7-7,5)$ & $(1,3-7,6)$ & $(5,3-11,1)$ & $(2,7-10,7)$ \\
p & \multicolumn{2}{c}{0,159} & & 0,049 & & 0,775 \\
\hline
\end{tabular}

$\mathrm{SM}=$ síndrome metabólico. Valores expresados en \% e intervalos de confianza.

Tabla 4. Asociación del SM y de sus componentes con inflamación en hombres

\begin{tabular}{|lcccccc|}
\hline & OR & PCR entre 3-10 & \multicolumn{3}{c|}{ PCR $>\mathbf{1 0}$} \\
SM & IC & p & OR & IC & p \\
PC elevado & 2,040 & $1,115-3,730$ & 0,021 & 0,865 & $0,176-4,260$ & 0,858 \\
\hline TG elevados & 3,866 & $2,043-7,314$ & 0,000 & 0,659 & $0,080-5,413$ & 0,698 \\
\hline HT & 2,049 & $1,187-3,539$ & 0,010 & 0,947 & $0,262-3,419$ & 0,934 \\
\hline HDL bajos & 1,066 & $0,617-1,843$ & 0,818 & 1,221 & $0,353-4,228$ & 0,753 \\
\hline Glicemia elevada & 1,343 & $0,729-2,473$ & 0,344 & 0,791 & $0,161-3,880$ & 0,773 \\
\hline
\end{tabular}

Modelo ajustado por NSE. Categoría de comparación: PCRus $<3$ mg/L. 
Tabla 5. Asociación del SM y de sus componentes con inflamación en mujeres

\begin{tabular}{|lcccccc|}
\hline & OR & PCR entre 3-10 & \multicolumn{3}{c|}{ PCR > 10 } \\
SM & IC & p & OR & IC & P \\
PC elevado & 1,118 & $0,740-1,690$ & 0,597 & 0,862 & $0,399-1,863$ & 0,705 \\
\hline TG elevados & 1,075 & $0,794-1,456$ & 0,640 & 2,649 & $1,201-5,844$ & 0,016 \\
HT & 1,285 & $0,847-1,949$ & 0,238 & 0,862 & $0,389-1,909$ & 0,641 \\
HDL bajos & 0,968 & $0,610-1,535$ & 0,889 & 1,281 & $0,589-2,780$ & 0,532 \\
\hline Glicemia elevada & 1,187 & $0,807-1,748$ & 0,384 & 1,022 & $0,515-2,031$ & 0,950 \\
\hline
\end{tabular}

Modelo ajustado por NSE. Categoría de comparación: PCRus $<3 \mathrm{mg} / \mathrm{L}$.

\section{Discusión}

En este estudio se pudo constatar que hubo asociación de SM con PCRus elevada y con algunos de los componentes del SM, específicamente con perímetro de cintura aumentado y triglicéridos altos, aunque sólo en hombres. En mujeres, la única asociación fue entre perímetro de cintura elevado y PCRus muy alta. Se pudo comprobar también que un tercio de las personas presentaban inflamación en grado moderado y $6,5 \%$ en valores altos.

Es interesante verificar que casi $40 \%$ de las personas estudiadas, adultos de mediana edad, presentan valores de PCRus sobre $3 \mathrm{mg} / \mathrm{L}$. Diversos estudios han señalado que, si bien la concentración de PCR aumenta rápidamente, a consecuencia de daño en los tejidos o infecciones, especialmente bacterianas (con valores superiores a $200 \mathrm{mg} / \mathrm{L}$ ) ${ }^{17}$, su utilidad como marcador de enfermedad cardiovascular también es ampliamente reconocido, ya que se ha descrito que valores de menor magnitud (>3 mg/L) se asocian a episodios isquémicos o infarto $^{18,19}$. Sin embargo, no hay unanimidad acerca de los niveles de PCR que se deben utilizar para predecir riesgo cardiovascular. Algunos autores utilizan los siguientes puntos de corte: bajo riesgo $(<1)$, intermedio (1-3), alto riesgo (3-10) y muy alto $(>10)$, en este último caso recomiendan repetir el examen o buscar otras patologías que estén explicando esta elevación ${ }^{16}$. Nosotros usamos una clasificación arbitraria, juntando riesgo bajo con intermedio, esperando que las asociaciones se dieran con la categoría de alto riesgo, que podría reflejar mejor la relación buscada.

Algunos estudios encuentran la asociación de SM con PCRus, empleando la definición más aceptada (presencia de 3 o más componentes), mientras que otros estudios reportan que los valores de PCR se elevan mientras más componentes estén presentes ${ }^{20-22}$.

Hay estudios que, a diferencia del presente trabajo, encuentran la relación en ambos sexos ${ }^{23}$. No debiera ser extraño encontrar diferencias en el comportamiento del SM por sexo, ya que si bien las mujeres tienen aparentemente mayor riesgo de tener SM (por la alta frecuencia de PC elevado y HDL bajos), los hombres tienen alto riesgo cardiovascular por mayor prevalencia de hipertensión arterial y triglicéridos elevados ${ }^{24}$. En la Encuesta Nacional de Salud, realizada en el país el 2009, se pudo comprobar parte de estas afirmaciones al encontrarse que los varones tienen mayor prevalencia de hipertensión arterial y triglicéridos altos, mientras que las mujeres tuvieron mayor frecuencia de HDL bajos ${ }^{6}$. La composición del grupo de estudio según sexo (y edad) determinará la mayor o menor frecuencia de factores de riesgo presentes, lo que está apoyado por la fisiopatología del SM en el que intervienen una compleja participación de factores biológicos, genéticos, hormonales y ambientales ${ }^{25}$. El que se haya encontrado una distribución diferente de PCRus elevada y presencia o no de SM, solamente en hombres (Tabla 3), es lo que apoya encontrar posteriormente una asociación de SM, como de algunos de sus componentes, con PCR en este rango de interés (Tabla 4).

Como el SM está definido por diversos componentes que ejercen su riesgo metabólico por distintas vías, no necesariamente asociadas a inflamación, el análisis de los factores de riesgo 
agrupados puede reflejar una condición metabólica diferente a la encontrada en el análisis de los componentes en forma individual. Esto, sumado a que los factores de riesgo que integran el SM aumentan con la edad, hace probable que las asociaciones se den más en personas mayores que en adultos más jóvenes, como los estudiados en esta oportunidad.

Lo que sí se ha reportado reiteradamente es la relación de obesidad con marcadores de inflamación, particularmente PCRus ${ }^{18,26}$ y reversibilidad de estos valores elevados al haber baja de peso ${ }^{27}$. Se ha descrito que en el exceso de peso, el tejido adiposo se ve expuesto a diferentes condiciones si el fenómeno se perpetúa: estrés oxidativo, estrés del retículo endoplasmático, hipoxia y reordenamiento masivo de la matríz extracelular, procesos que funcionan como señal de alerta. El tejido adiposo envía señales producto de la exposición continua a diversos estímulos, principalmente adscritos a la secreción de la proteína quimioatractante de macrófagos (MCP-1). Esta molécula induce la migración y posterior infiltración de macrófagos al tejido adiposo, estableciendo un estado de inflamación crónica de bajo grado. Estas células interactúan con los adipocitos residentes y en conjunto aumentan la secreción (local y sistémica) de adipoquinas y citoquinas pro-inflamatorias (resistina, leptina, TNFa, IL-6 entre otras) y disminución de moléculas antiinflamatorias (adiponectina). Esta inflamación puede ser pesquisada en la periferia a través de la detección de cada uno de estos metabolitos o de moléculas activadas secundariamente, como es el caso de la $\mathrm{PCR}^{28}$.

En nuestro estudio, al igual que lo reportado en otros trabajos ${ }^{29}$, se obtuvo que el perímetro de cintura aumentado es el principal determinante de la elevación de la PCRus en el síndrome metabólico.

Hay estudios que han señalado una asociación directa entre PCRus con triglicéridos e inversa con HDL, pero también reportan que el IMC, o la obesidad es la variable más relacionada con inflamación en bajo grado ${ }^{26,30}$. Respecto a la asociación con hipertensión arterial, también hay estudios que señalan que la PCR está elevada en pacientes hipertensos ${ }^{31,32}$ y que este proceso inflamatorio, presente en la fisiopatología de la hipertensión, predice el desarrollo de enfermedad cardiovascular, sin embargo, en este estudio no se encontró esta asociación.
A pesar de que este es un estudio poblacional, con personas relativamente sanas, se encontró que $7 \%$ tiene valores de PCRus $>10 \mathrm{mg} / \mathrm{L}$. Este hallazgo llama la atención y merece mayor estudio. Se ha descrito que estos valores tan elevados se encuentran en patologías virales, enfermedades inflamatorias ${ }^{33}$ o cáncer $^{34-36}$. La justificación de incorporarlas en el análisis fue para ver si un mayor grado de inflamación se asociaba a factores de riesgo metabólico, aspecto que no se encontró, salvo su asociación con obesidad abdominal en mujeres.

En este estudio se demostró, también, que en esta población aparentemente sana y joven existe alta prevalencia de SM y de los componentes que lo integran. Estos datos son similares a lo reportado en la Encuesta Nacional de Salud, en que el grupo de 25-44 años tiene 27,5\% de SM, aunque en esta encuesta nacional, la prevalencia fue mayor en varones $^{37}$. Puede llamar la atención, en nuestro estudio, la elevada frecuencia de personas con glicemia $>100 \mathrm{mg} / \mathrm{dL}$, lo que también es concordante con datos nacionales. La ENS 2009 indica $9,4 \%$ de personas con glicemia $\geq 126 \mathrm{mg} / \mathrm{dL}$ (en nuestro estudio es $3,1 \%$ ). Si restaramos a $23,6 \%$ de valores $>100 \mathrm{mg} / \mathrm{dL}, 3,1 \%$ que tiene glicemia $\geq 126 \mathrm{mg} / \mathrm{dL}$, queda $20 \%$ de personas cuyas glicemias estarían entre 100 y $126 \mathrm{mg} / \mathrm{dL}$. En la ENS 2003 (encuesta realizada 8 años antes que nuestro estudio) este valor corresponde a $15,6 \%$ (dato que no está publicado en la ENS 2009). Considerando además que el exceso de peso es cada vez más frecuente ( $75 \%$ en nuestra muestra) y que se asocia a hiperglicemia, es bastante probable que los valores más actuales de glicemia $>100$ estén en los valores por nosotros reportados.

Entre las fortalezas de este estudio está disponer de gran número de personas en comunas semi-rurales, en un rango de edad estrecho y que pertenecen a un NSE medio a medio bajo, aunque, esta relativa homogeneidad también se puede interpretar como una debilidad muestral, al no tener personas de más edad en los que la frecuencia de SM (y sus componentes) sea mayor o una muestra que exprese mejor la variabilidad socioeconómica poblacional.

El encontrar a esta edad alta frecuencia de factores de riesgo asociados a inflamación es una indicación de la importancia de realizar estos estudios en personas que aún no tienen las enfermedades que producen discapacidad y muerte y que proporcionan insumos para la vigilancia 
epidemiológica y para fortalecer los programas dirigidos a la prevención y control de enfermedades crónicas, como la obesidad, desde temprana edad. Finalmente, los resultados de este estudio entregan antecedentes para la vigilancia de la población de adultos de mediana edad en los servicios de salud, para evitar las consecuencias que puedan tener estos factores de riesgo, con mensajes dirigidos a la población en riesgo, que según los resultados de este estudio, corresponde a hombres adultos de mediana edad.

\section{Referencias}

1. Zhou C. Lung cancer molecular epidemiology in China: recent trends. Transl Lung Cancer Res 2014; 3 (5): 2709.

2. Baena R, Salinas P. Diet and colorectal cancer. Maturitas 2015; 80 (3): 258-64.

3. Kohli P. Primary and secondary prevention of ischemic heart disease in women. Curr Atheroscler Rep 2015; 17 (7): 518.

4. Lastra G, Manrique C. Perivascular adipose tissue, inflammation and insulin resistance: link to vascular dysfunction and cardiovascular disease. Horm Mol Biol Clin Investig 2015; 22 (1): 19-26.

5. Pietri P, Vlachopoulos C, Tousoulis D. Inflammation and Arterial Hypertension: From Pathophysiological Links to Risk Prediction. Curr Med Chem 2015; 22 (23): 2754-61.

6. MINSAL. Encuesta nacional de Salud, 2010. Available from: http://web.minsal.cl/portal/url/item/bcb03d7bc28b64dfe040010165012d23pdf. [Consultado en marzo de 2016].

7. Devran O, Karakurt Z, Adiguzel N, Gungor G, Mocin $\mathrm{OY}$, Balci MK, et al. C-reactive protein as a predictor of mortality in patients affected with severe sepsis in intensive care unit. Multidiscip Respir Med 2012; 7 (1): 47.

8. Jeschke MG, Finnerty CC, Kulp GA, Kraft R, Herndon DN. Can we use C-reactive protein levels to predict severe infection or sepsis in severely burned patients? Int J Burns Trauma 2013; 3 (3): 137-43.

9. Bluher M. Adipose tissue inflammation: a cause or consequence of obesity-related insulin resistance? Clin Sci (Lond) 2016; 130 (18): 1603-14.

10. Galic S, Oakhill JS, Steinberg GR. Adipose tissue as an endocrine organ. Mol Cell Endocrinol 2010; 316 (2): 129-39.

11. Vio F, Albala C, Kain J. Nutrition transition in Chile revisited: mid-term evaluation of obesity goals for the period 2000-2010. Public Health Nutr 2008; 11 (4): 405-12.

12. Garmendia ML, Alonso FT, Kain J, Uauy R, Corvalán C. Alarming weight gain in women of a post-transitional country. Public Health Nutr 2014; 17 (3): 667-73.

13. Burrows R, Atalah E, Leiva L, Rojas P, Maza MP, Vásquez $\mathrm{F}$, et al. [Metabolic syndrome prevalence in Chilean children and adolescent with family history of chronic noncommunicable diseases]. Arch Latinoam Nutr 2012; 62 (2): 155-60.

14. Executive Summary of The Third Report of The National Cholesterol Education Program (NCEP) Expert Panel on Detection, Evaluation, And Treatment of High Blood Cholesterol In Adults (Adult Treatment Panel III). JAMA 2001; 285 (19): 2486-97.

15. ADIMARK. Manual de Aplicación Encuesta Socioeconómica 2000. Available from: http://www.microweb. cl/idm/documentos/ESOMAR.pdf [Consultado en noviembre de 2015].

16. Yeh ET, Willerson JT. Coming of age of C-reactive protein: using inflammation markers in cardiology. Circulation 2003; 107 (3): 370-1.

17. Ansar W, Ghosh S. C-reactive protein and the biology of disease. Immunol Res 2013; 56 (1): 131-42.

18. Ganguli D, Das N, Saha I, Sanapala KR, Chaudhuri $\mathrm{D}$, Ghosh S, et al. Association between inflammatory markers and cardiovascular risk factors in women from Kolkata, W.B, India. Arq Bras Cardiol 2011; 96 (1): 3846.

19. Koenig W. High-sensitivity C-reactive protein and atherosclerotic disease: from improved risk prediction to risk-guided therapy. Int J Cardiol 2013; 168 (6): 512634.

20. Farooq W, Farwa U, Khan FR. The metabolic syndrome and inflammation: role of insulin resistance and increased adiposity. Oman Med J 2015; 30 (2): 100-3.

21. Sur G, Floca E, Kudor-Szabadi L, Sur ML, Sur D, Samasca G. The relevance of inflammatory markers in metabolic syndrome. Maedica (Buchar) 2014; 9 (1): 15-8.

22. Lee K, Song YM, Sung J. Genetic and environmental associations between C-reactive protein and components of the metabolic syndrome. Metab Syndr Relat Disord 2013; 11 (2): 136-42.

23. Lai MM, Li CI, Kardia SL, Liu CS, Lin WY, Lee YD, et al. Sex difference in the association of metabolic syndrome with high sensitivity C-reactive protein in a Taiwanese population. BMC Public Health 2010; 10: 429.

24. Rochlani Y, Pothineni NV, Mehta JL. Metabolic Syndrome: Does it Differ Between Women and Men? Cardiovasc Drugs Ther 2015; 29 (4): 329-38. 
25. Wu DM, Chu NF, Shen MH, Wang SC. Obesity, plasma high sensitivity $\mathrm{C}$-reactive protein levels and insulin resistance status among school children in Taiwan. Clin Biochem 2006; 39 (8): 810-5.

26. Tchernof A, Nolan A, Sites CK, Ades PA, Poehlman ET. Weight loss reduces C-reactive protein levels in obese postmenopausal women. Circulation 2002; 105 (5): 564-9.

27. Wellen KE, Hotamisligil GS. Obesity-induced inflammatory changes in adipose tissue. J Clin Invest 2003; 112 (12): 1785-8.

28. Nakamura H, Ito H, Egami $Y$, Kaji Y, Maruyama T, Koike $\mathrm{G}$, et al. Waist circumference is the main determinant of elevated C-reactive protein in metabolic syndrome. Diabetes Res Clin Pract 2008; 79 (2): 330-6.

29. Izaola O, De Luis D, Sajoux I, Domingo JC, Vidal M. [Inflammation and obesity (lipoinflammation)]. Nutr Hosp 2015; 31 (6): 2352-8.

30. Schillaci G, Pirro M. C-reactive protein in hypertension: clinical significance and predictive value. Nutr Metab Cardiovasc Dis 2006; 16 (7): 500-8.

31. Savoia C, Schiffrin EL. Inflammation in hypertension. Curr Opin Nephrol Hypertens 2006; 15 (2): 152-8.

32. Karadeniz G, Polat G, Senol G, Buyuksirin M. C-reactive protein measurements as a marker of the severity of chronic obstructive pulmonary disease exacerbations. Inflammation 2013; 36 (4): 948-53.

33. Xu M, Zhu M, Du Y, Yan B, Wang Q, Wang C, et al. Serum C-reactive protein and risk of lung cancer: a case-control study. Med Oncol 2013; 30 (1): 319.

34. McSorley MA, Alberg AJ, Allen DS, Allen NE, Brinton LA, Dorgan JF, et al. C-reactive protein concentrations and subsequent ovarian cancer risk. Obstet Gynecol 2007; 109 (4): 933-41.

35. Guo YZ, Pan L, Du CJ, Ren DQ, Xie XM. Association between $\mathrm{C}$-reactive protein and risk of cancer: a meta-analysis of prospective cohort studies. Asian Pac J Cancer Prev 2013; 14 (1): 243-8. 